\title{
Tackling Instrumental Activities of Daily Living: Time to Start Rethinking Items
}

\author{
Soong-nang Jang \\ Red Cross College of Nursing, Chung-Ang University, Seoul, Korea
}

I read with great pleasure the Letter to the Editor by Kim and Won ${ }^{1)}$ regarding our study ${ }^{2)}$ as the authors are dedicated researchers who develop instruments and pursue important issues concerning functional status and frailty measurements in Korea. I respect these scholars and have used the Korean versions of the Activities of Daily Living (K-ADL) and Instrumental Activity of Daily Living (K-IADL) scales developed by Dr. Won in my previous studies on disability. The motivation for our item response theory (IRT) research was prompted during international comparative studies on disability rates. Each country has somewhat different IADL items and differential item functioning (DIF) even for the same items. Thus, there might be potential measurement biases in disability comparisons across international surveys of aging. ${ }^{3)}$ Without considering DIF, the disability rates of specific societies may not be free with possible misinterpretation. However, we realized that IRT analyses of the K-ADL and K-IADL were lacking.

Regimes, politics, and policies are ultimately rooted in the psychosocial background of a society such that the social-cultural context cannot be easily changed. ${ }^{4)}$ In terms of understanding cultural differences, empirical analysis results should be accumulated more closely. Identifying a policy direction that fits the cultural and practical perspectives will ensure compliance and sustainability of healthy aging policies. To better understand how societies' care for older adults works, it is necessary to expand institutional variation and conduct international comparative studies as a wider scope of political, policy, and social elements to confirm the relevance that allows a clearer association between individual and social factors for healthy aging.

Items in certain scales might be biased with respect to difficulty and/or discrimination. Item bias is the degree to which the items comprising a measurement scale are systematically related to various exogenous variables (e.g., age, gender, and socioeconomic position) after conditioning for the latent variable of interest. Item difficulty bias occurs when one group, such as women, responds higher on a certain item after being matched to men on the total scale score. Item discrimination bias occurs when the item difficulty bias (between two groups matched for the latent variable) increases or decreases as a function of the level of the latent variable. ${ }^{5)}$

If the current Korean older generations, such as baby boomers, progress to "gender egalitarians" (as Kim \& Won ${ }^{1)}$ mentioned), it is reasonable to consider changing some of the response options and items in the K-IADL. For example, "household chores" and "preparing meals" require consideration regarding whether response options, such as "never have done before" or "don't do even though I can", have to be maintained only because they are "men". Many Korean researchers have excluded three items related to household tasks (household chores, preparing meals, and doing laundry) from the K-IADL in their analyses of men because of these niggling additional responses. However, for analysis, whether it remains valid and justifiable to remove items corresponding to household tasks only in men requires further evaluation. I would like to specifically ask the following research question: which items should be excluded from men, and which items should be excluded from women and why? My aim was not to assess gender differences in the status of disability for each ADL and IADL item but rather to examine the reliability of the scales according to gender.

Our findings indicated that only two items in the K-IADL (Grooming and Take medications) did not show a response bias. ${ }^{2)}$ The IADLs were originally designed to assess a more complex range of functioning, in that they require more skill, judgment, and independence than those required for ADLs. They measure an individual's functioning in the social world and the world outside the home. Given this, can we achieve the value of IADLs with only two valid items? Item bias analyses often use a "purified subscale" method, ${ }^{6)}$ in which the purified subscale is defined as the total scale score minus the biased items. Our study was performed to as- 
sess whether the choice of refined scale is clear in real data. We believe this is the beginning: the ultimate implication is that additional research is needed on how to improve K-IADL items.

Finally, it is important to have a clear conceptual framework for any measure. ADLs and IADLs are useful for measuring progress toward healthcare goals and encouraging geriatricians caring for patients to shift their attention toward providing comprehensive help for their older patients with disabilities. ${ }^{7)}$ For this fundamental value of ADLs and IADLs, we can describe "disability" according to "how much help is needed" in the K-ADL and K-IADL. The IADL scale fits well for disability when qualifying phrases concerning social and cultural factors are included.

It may not be possible to completely control for bias in all measurements. However, the establishment of item invariance is of prime importance for drawing unbiased inferences in gerontological research using multi-item functional status measurements. The results have methodological and substantive implications for the suitable use of IADL for all ages, genders, and socioeconomic positions. There is a need to rethink the K-IADL for comprehensive geriatric care and long-term care services. Improved measurements may help clarify scientific evidence for the development of policies toward healthy aging societies.

\section{ACKNOWLEDGMENTS}

\section{CONFLICT OF INTEREST}

The author claims no conflicts of interest.

\section{REFERENCES}

1. Kim S, Won CW. How can we evaluate disability without bias? Ann Geriatr Med Res 2020;24:152-3.

2. Jang SN, Kawachi I. Why do older Korean adults respond differently to activities of daily living and instrumental activities of daily living? A differential item functioning analysis. Ann Geriatr Med Res 2019;23:197-203.

3. Chan KS, Kasper JD, Brandt J, Pezzin LE. Measurement equivalence in $\mathrm{ADL}$ and IADL difficulty across international surveys of aging: findings from the HRS, SHARE, and ELSA.J Gerontol B Psychol Sci Soc Sci 2012;67:121-32.

4. Krieger N. Epidemiology and the people's health: theory and context. New York, NY: Oxford University Press; 2011.

5. Holland PW, Wainer H. Differential item functioning: theory and practice. Hillsdale, NJ: Lawrence Erlbaum Associates; 1993.

6. Dean K, Salem N. Detecting measurement confounding in epidemiological research: construct validity in scaling risk behaviours: based on a population sample in Minnesota, USA. J Epidemiol Community Health 1998;52:195-9.

7. Kovar MG, Powell Lawton M. Functional disability: activities and instrumental activities of daily living. Annu Rev Gerontol Geriatr 1994;14:57-75.

Corresponding Author: Soong-nang Jang, $\mathrm{PhD}$

Red Cross College of Nursing, Chung-Ang University, 84, Heukseok-ro,

Dongjak-gu, Seoul 06974, Korea

E-mail: sjang@cau.ac.kr

ORCID: https://orcid.org/0000-0003-2621-945X

Received: August 22, 2020; Accepted: August 26, 2020 
